\title{
Quantitative analysis of viral RNA kinetics in coxsackievirus B3-induced murine myocarditis: biphasic pattern of clearance following acute infection, with persistence of residual viral RNA throughout and beyond the inflammatory phase of disease
}

\author{
K. Nundita Reetoo, Shabina A. Osman, Shirin J. Illavia, Charlotte L. Cameron-Wilson, \\ Jangu E. Banatvala and Peter Muir
}

Department of Infection, Guy's, King's \& St Thomas' School of Medicine, King's College London, St Thomas' Campus, Lambeth Palace Road, London SE1 7EH, UK

\begin{abstract}
Although the association remains controversial, enteroviruses have been implicated in the aetiology of several chronic diseases of humans. To further understand the mechanism of enterovirus persistence and its relationship to organ pathology, virus infectivity and viral RNA kinetics in the heart and other target organs during acute and persistent phases of murine coxsackievirus B3 infection were investigated. These studies revealed a biphasic pattern of virus clearance. Thus, there was a rapid but incomplete clearance of viral RNA from the myocardium following the acute phase of virus replication, which paralleled the elimination of virus infectivity. The mean half-life of viral RNA between days 5 and 14 post-infection (p.i.) was $13.4 \mathrm{~h}$. In contrast, a much slower rate of decline in viral RNA levels was observed during the post-infectious inflammatory phase of myocarditis. The mean half-life of viral RNA between days 14 and 90 p.i. was 14.1 days. Viral RNA persisted in the myocardium beyond the resolution of inflammation and was still detectable in a proportion of animals 90 days after infection. Clearance of viral RNA from other target organs occurred more rapidly, but the rate of clearance was largely independent of the level of viral RNA present during the acute phase of infection. Thus, while antiviral immune responses effectively eliminated infectious virus, clearance of residual viral RNA from the myocardium and other target organs was significantly delayed, despite a prolonged inflammatory response. These findings suggest that clearance of persistent enterovirus infection requires mechanisms different from those responsible for the elimination of virus infectivity.
\end{abstract}

\section{Introduction}

Group B coxsackieviruses (CVB) consist of six serotypes within the enterovirus genus Picornaviridae, a large family of single-stranded positive-sense RNA viruses. They are associated with a wide spectrum of human illness ranging in severity from subclinical infection to rapidly fatal disease, with symptoms as diverse as acute myocarditis, aseptic meningitis, encephalitis, Bornholm disease (epidemic pleurodynia), nonspecific rashes and febrile illness. Although classically regarded as agents of acute self-limiting infections, a considerable body of evidence also implicates CVB and other enteroviruses in the aetiology of a number of chronic diseases, including dilated

Author for correspondence: Peter Muir.

Fax +44 207922 8387. e-mail peter.muir@kcl.ac.uk cardiomyopathy, possibly type 1 (insulin-dependent) diabetes mellitus (IDDM), and chronic fatigue syndrome. However, infectious virus cannot be isolated from patients with chronic disease and the persistence of enteroviral RNA in tissue from an immunologically normal host is a concept that challenges our current understanding of enterovirus biology (Modlin, 2000). Furthermore, the pathogenesis of at least some chronic enterovirus-associated diseases involves an autoimmune component, which may explain chronic pathology in the absence of virus persistence. Consequently there is a lack of agreement among investigators as to the role of enterovirus persistence in these chronic diseases (reviewed by Melchers et al., 1994; Muir \& Archard, 1994).

A number of investigators have developed murine models of picornavirus-induced disease, such as myocarditis (Grodums \& Dempster, 1959; Woodruff \& Kilbourne, 1970), IDDM 
(Webb et al., 1976; Yoon et al., 1978), poliomyelitis (Jubelt et al., 1980; Ren et al., 1991), polymyositis (Strongwater et al., 1984) or chronic demyelinating disease (Lipton, 1975). These models have been used to study the natural course of infection and immunity, and the mechanisms of pathogenesis. Numerous studies have documented virus persistence beyond the acute phase of infection in mice. These persistent infections can be classified as either productive, where infectious virus is detectable continuously or intermittently during the chronic phase of infection (Lipton \& Dal Canto, 1976; Miller, 1981), or non-productive, where infectious virus cannot be isolated beyond the acute phase of infection, but viral RNA can nonetheless be detected using molecular hybridization or amplification techniques (Destombes et al., 1997; Klingel et al., 1992; Kyu et al., 1992; Tam et al., 1991). Persistent productive enterovirus has been observed in patients with gammaglobulin deficiency (reviewed by McKinney et al., 1987). However, the second pattern of non-productive enterovirus persistence appears similar to that reported in immunocompetent humans with chronic enterovirus-associated disease. The molecular mechanism of enterovirus persistence in the absence of productive infection, the extent of viral genome expression, and its possible consequences for the infected cell or organism are not fully understood.

The duration for the persistence of the virus genome is likely to be determined by the rates of residual viral RNA replication, catabolic degradation of viral RNA and virus- or immune-mediated cytolysis of infected cells. We considered that quantitative analysis of viral RNA kinetics would shed light on the net effect of these mechanisms on the fate of viral RNA in a model of persistent picornavirus infection. Therefore, we studied viral RNA kinetics in acute and chronic phases of CVB3-induced murine myocarditis using quantitative-competitive PCR (Reetoo et al., 1999). Viral RNA levels in the myocardium were quantified over a 90 day period following initiation of infection. Viral RNA persistence was also investigated in other target organs of CVB3 infection. For these studies we used the SWR $\left(H-2^{q}\right)$ mouse strain, which is highly susceptible to both acute and chronic CVB3-induced myocarditis but shows little or no morbidity or mortality (Klingel et al., 1992; Zhang et al., 1994) and is, therefore, suitable for studying persistent infection. This approach yielded valuable insight into the rate of viral RNA clearance during acute and persistent phases of CVB3 infection in different target organs and its temporal relationship to inflammatory responses.

\section{Methods}

- Cells and viruses. CVB3 virus (Nancy strain) was originally obtained from R. Kandolf (Tübingen) (Kandolf \& Hofschneider, 1985) and was propagated in Vero cells. The method of Reed \& Muench (1938) was used to calculate the $\mathrm{TCID}_{50}$.

Murine model of CVB3 myocarditis. Inbred male four-week-old SWR mice (Harlan) were inoculated intraperitoneally with $10^{6} \mathrm{TCID}_{50}$
CVB3 in $100 \mu \mathrm{l}$ PBS. Mice inoculated with $100 \mu \mathrm{l}$ PBS served as uninfected controls. Internal organs, whole EDTA-anticoagulated blood and serum were aseptically collected from four uninfected mice at day 0 post-infection (p.i.), and from infected mice (four or five) and uninfected mice (two or three) at time-points from day 3 to day 90 p.i. Organs were washed in sterile PBS and a portion was snap-frozen in liquid nitrogen and stored at $-70{ }^{\circ} \mathrm{C}$ for virus isolation and PCR analysis. The remainder was fixed in formal-buffered saline for histological studies. Serum was also stored at $-70^{\circ} \mathrm{C}$, but whole blood was processed immediately for virus infectivity and PCR analysis as described below.

Titration of virus infectivity in murine myocardium. Preweighed portions of approximately $10 \mathrm{mg}$ cryopreserved tissue were ground to a fine powder in liquid nitrogen, homogenized in $200 \mu$ l sterile PBS, filtered through a $0.45 \mu \mathrm{m}$ pore size and serially diluted in PBS. Replicate aliquots $(100 \mu \mathrm{l})$ of serial tenfold dilutions were inoculated onto Vero cell monolayers in microwell cultures. After 7 days incubation at $37{ }^{\circ} \mathrm{C}$ in a $5 \% \mathrm{CO}_{2}$ atmosphere, infectivity titres were determined and expressed as $\mathrm{TCID}_{50} / \mathrm{mg}$ tissue. Whole blood samples and filtered serum samples were cultured undiluted; the inoculum was aspirated after $24 \mathrm{~h}$ and replaced with $100 \mu \mathrm{l}$ maintenance medium.

Qualitative enterovirus PCR. Total RNA was extracted from whole blood and serum using RNAzol B (Biogenesis) and from cryopreserved tissue after grinding in liquid nitrogen and homogenization in RNAzol B. The presence of enterovirus RNA was tested by qualitative nested RT-PCR (EV nPCR) as described previously (Nicholson et al., 1994). In titration experiments using synthetic RNA transcripts corresponding in sequence to the $5^{\prime}$ nontranslated region of the CVB3 genome (Reetoo et al., 1999), this assay was found to have a lower detection level of 10 to 100 genome equivalents.

Quantitative enterovirus PCR (EV qPCR). CVB3 RNA in murine tissues was quantified by EV qPCR as described previously (Reetoo et al., 1999). Briefly, total RNA in $100 \mu \mathrm{l}$ whole blood or serum, or pre-weighed portions of approximately $10 \mathrm{mg}$ cryopreserved tissue was co-extracted and co-amplified with $10^{4}$ copies of an internal standard synthetic RNA transcript (IS RNA). The CVB3 target sequence of the EV qPCR assay, with a modified internal probe recognition sequence to allow differentiation of IS RNA-derived PCR product from CVB3 RNAderived product, was contained within the IS RNA sequence. The ratio of CVB3-derived and IS RNA-derived PCR product was then determined by hybridization with specific ruthenium-labelled probes and electrochemiluminescent quantification using QPCR 5000 (Perkin Elmer/ Applied Biosystems). From this ratio, the CVB3 RNA copy number in the original sample was determined by reference to a standard curve generated from EV qPCR analysis of increasing copy numbers of a synthetic transcript corresponding to nucleotides 1 to 645 of the CVB3 genome. The use of this standard curve to determine viral RNA copy number accounts for any difference in the amplification efficiencies of the IS RNA and CVB3 RNA templates. Where high levels of viral RNA in the test sample resulted in complete inhibition of IS RNA amplification, samples were retested in the presence of higher IS RNA copy numbers to allow accurate quantification. For samples in which the amplification of either CVB3 RNA or IS RNA failed, RNA was reprecipitated in ethanol and washed three times with $70 \%$ ethanol, prior to repeating EV qPCR in an effort to remove possible enzymatic inhibitors. The EV qPCR assay has been previously shown to be able to quantify as little as 100 copies of viral RNA/mg tissue, or per $10 \mu \mathrm{l}$ whole blood or serum (Reetoo et al., 1999). All samples were tested in duplicate and the mean values obtained were used in subsequent analyses. Blank RNA extractions, in which sterile $\mathrm{H}_{2} \mathrm{O}$ was processed in place of tissue, and PCR reagent blanks, in 

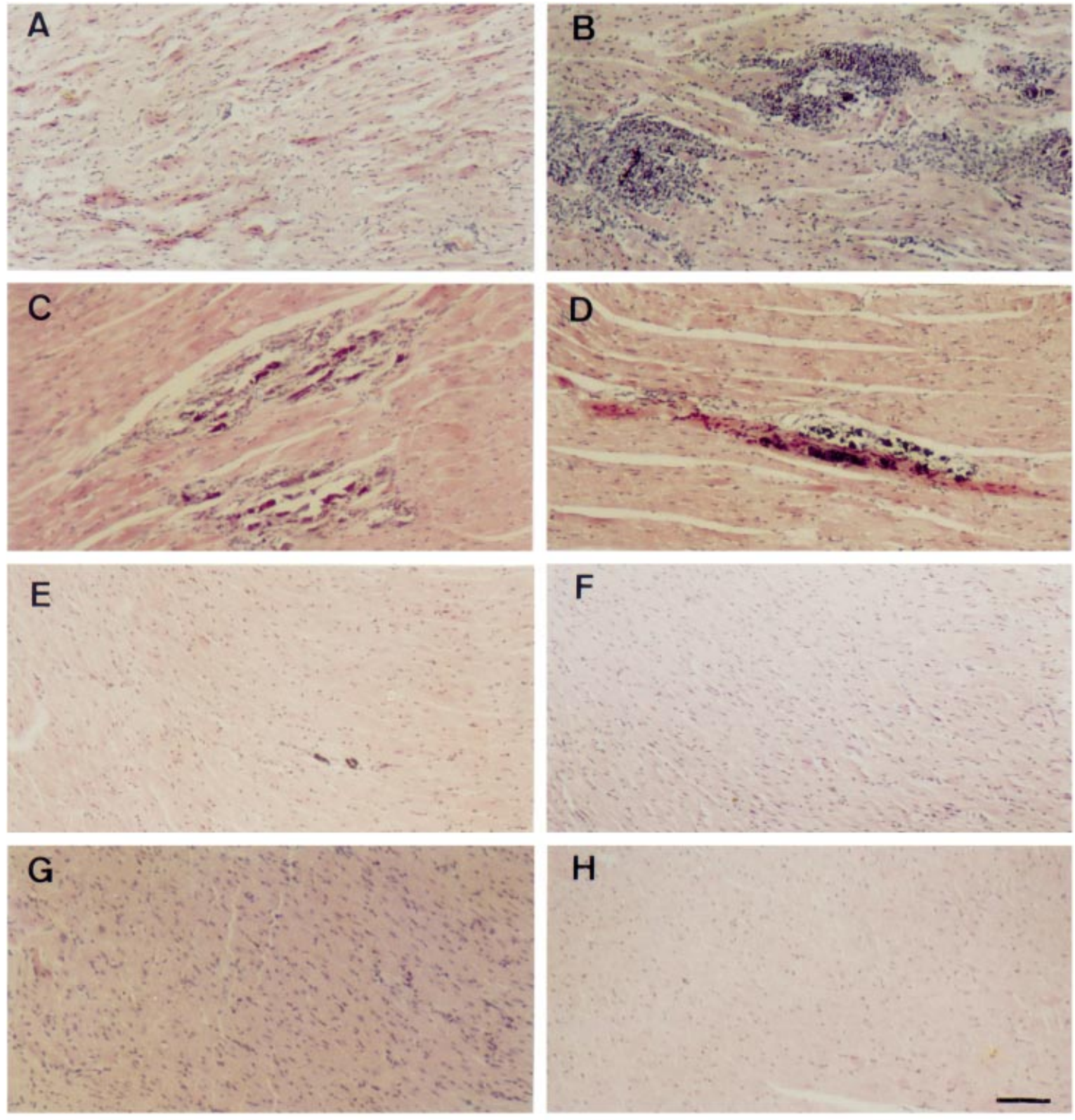

H
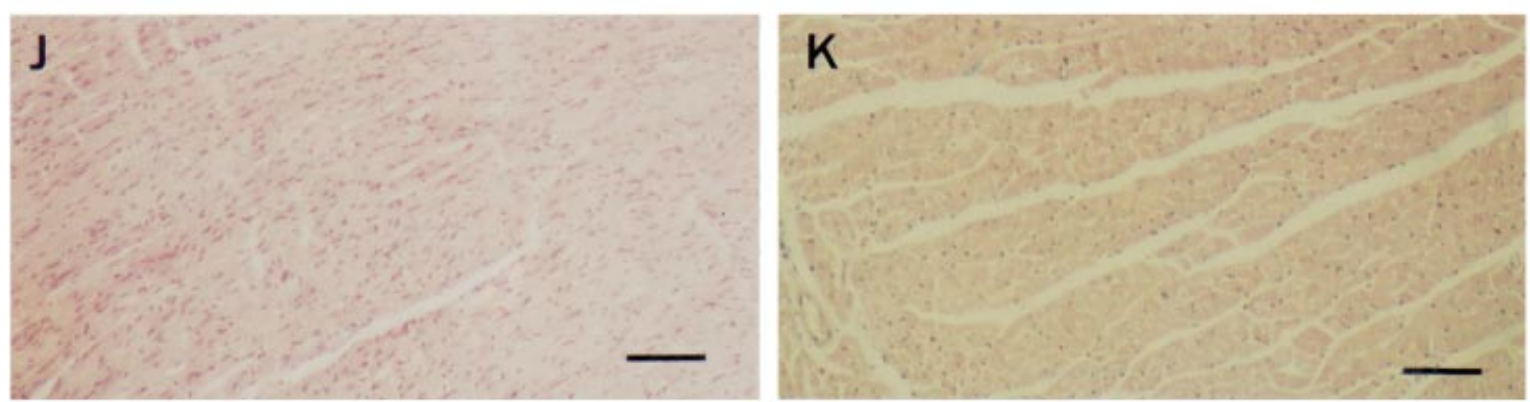

Fig. 1. Myocardial inflammation and calcium deposition in CVB3-infected $(A-E)$ and uninfected $(F-K)$ myocardium collected on days $3(A, F), 7(B, G), 48(C, H), 70(D, J)$ and $90(E, K)$ p.i. Solid bar indicates $100 \mu \mathrm{m}$.

which sterile $\mathrm{H}_{2} \mathrm{O}$ was added to EV qPCR reagents in place of test RNA extract, were included in each test batch to exclude the possibility of PCR contamination.
Histology. Tissue portions were fixed in formal-buffered saline and embedded in paraffin. Sections were stained with haematoxylin and eosin and observed by light microscopy. Inflammatory lesions were 
enumerated blind with respect to the infection status of individual animals or to the time after infection at which tissues were collected.

Data analysis. Viral RNA levels and virus infectivity in murine tissues were calculated as described previously (Reed \& Muench, 1938; Reetoo et al., 1999) and assembled using Microsoft Excel (1997). Mean logarithmic values and $95 \%$ confidence intervals were determined for each time-point. Trendlines obtained by linear regression were employed to determine mean rates of viral RNA clearance during different phases of infection and to estimate the time required for complete clearance of viral RNA (i.e. $<1$ viral genome/mg tissue).

\section{Results}

\section{Natural progression of CVB3-induced myocarditis}

Mice were observed daily for morbidity and mortality during the course of these experiments. In keeping with previous studies using this model of myocarditis (Zhang et al., 1994), there was no apparent morbidity and no mortality during the course of infection. Histological examination of myocardial tissue revealed the presence of focal inflammatory infiltrates as early as day 3 p.i. (Fig. I A). Inflammation was maximal on days 7 to 21 p.i. (Fig. 1B) and gradually subsided thereafter. Resolving lesions, showing extensive calcification and persisting residual inflammation are shown in Fig. $\mathrm{I}(\mathrm{C})$, and focal lesions were still present as late as day 70 p.i. (Fig. 1D). Inflammation was not observed in hearts beyond day 70 p.i., although residual calcium deposits were still present (Fig. IE). Myocardial inflammation was observed in 51 of 52 CVB3infected animals between days 3 and 70 p.i.; the heart of one CVB3-infected animal collected on day 35 p.i. appeared histologically normal, as did all 28 hearts from uninfected controls (Fig. 1F-K).

\section{Virus infectivity and viral RNA levels during acute and chronic phases of murine CVB3 infection}

The kinetics of CVB3 infection in the murine heart during acute and chronic phases of myocarditis were studied by quantifying virus infectivity and viral RNA levels in the myocardium at various time-points from day 0 to day 90 p.i. during the course of virus infection. Viral RNA levels in whole blood and serum collected on days 0 to 48 p.i. were also quantified in order to identify the viraemic phase of acute infection. EV qPCR analysis was successful in all but three of 52 myocardial samples (one collected on day 21 p.i. and two collected on day 42 p.i.) and three whole blood samples (collected on days 0,21 and 42 p.i.), in which test failure occurred despite RNA reprecipitation. These samples were therefore excluded from the analysis of viral RNA levels. Virus infectivity and viral RNA levels in the myocardium peaked at day 3 p.i. then declined. Virus infectivity showed a mean daily reduction in titre of $\log 0.828$ between days 3 and 7 p.i. $\left(r^{2}=\right.$ 0.716 ), corresponding to a half-life of $8.7 \mathrm{~h}$, and was eliminated by day 14 p.i. (Fig. 2). In contrast, clearance of viral RNA was biphasic; while the rate of clearance was initially similar to that of virus infectivity, residual viral RNA persisted at a low level throughout the duration of the 90 day observation period, with only a modest decline in levels between day 14 and day 90 p.i. (Fig. 2). Viral RNA levels showed a mean daily reduction in copy number of $\log 0.540$ between days 5 and 14 p.i. $\left(r^{2}=\right.$ 0.942 ), corresponding to a viral RNA half-life of $13.4 \mathrm{~h}$. Complete clearance of viral RNA, had it continued unabated at this rate, would have occurred by day 21 p.i. However, viral RNA persisted in the myocardium at detectable levels ( $>100$

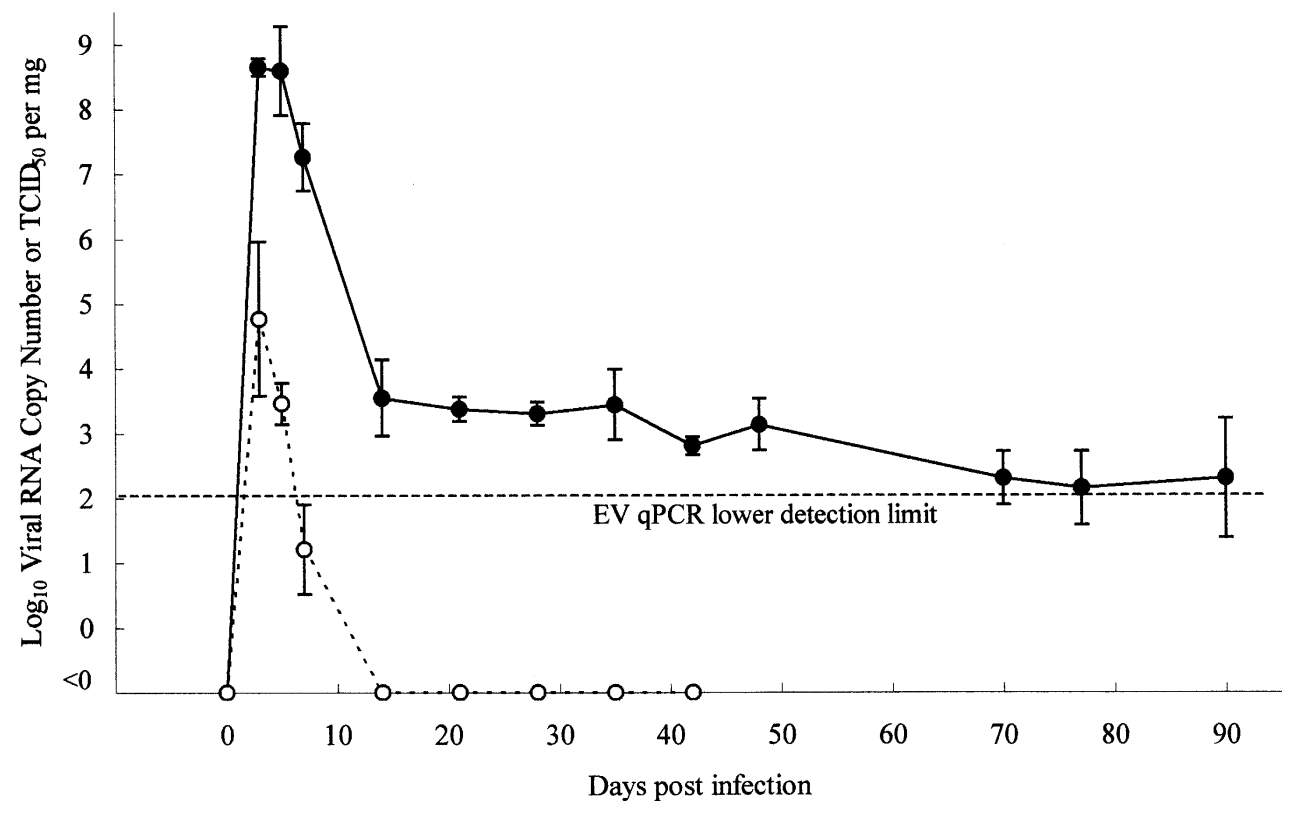

Fig. 2. Mean virus infectivity $(O)$ and viral RNA levels $(\bullet)$ in the myocardium collected from CVB3-infected mice. Error bars represent $95 \%$ confidence intervals. 


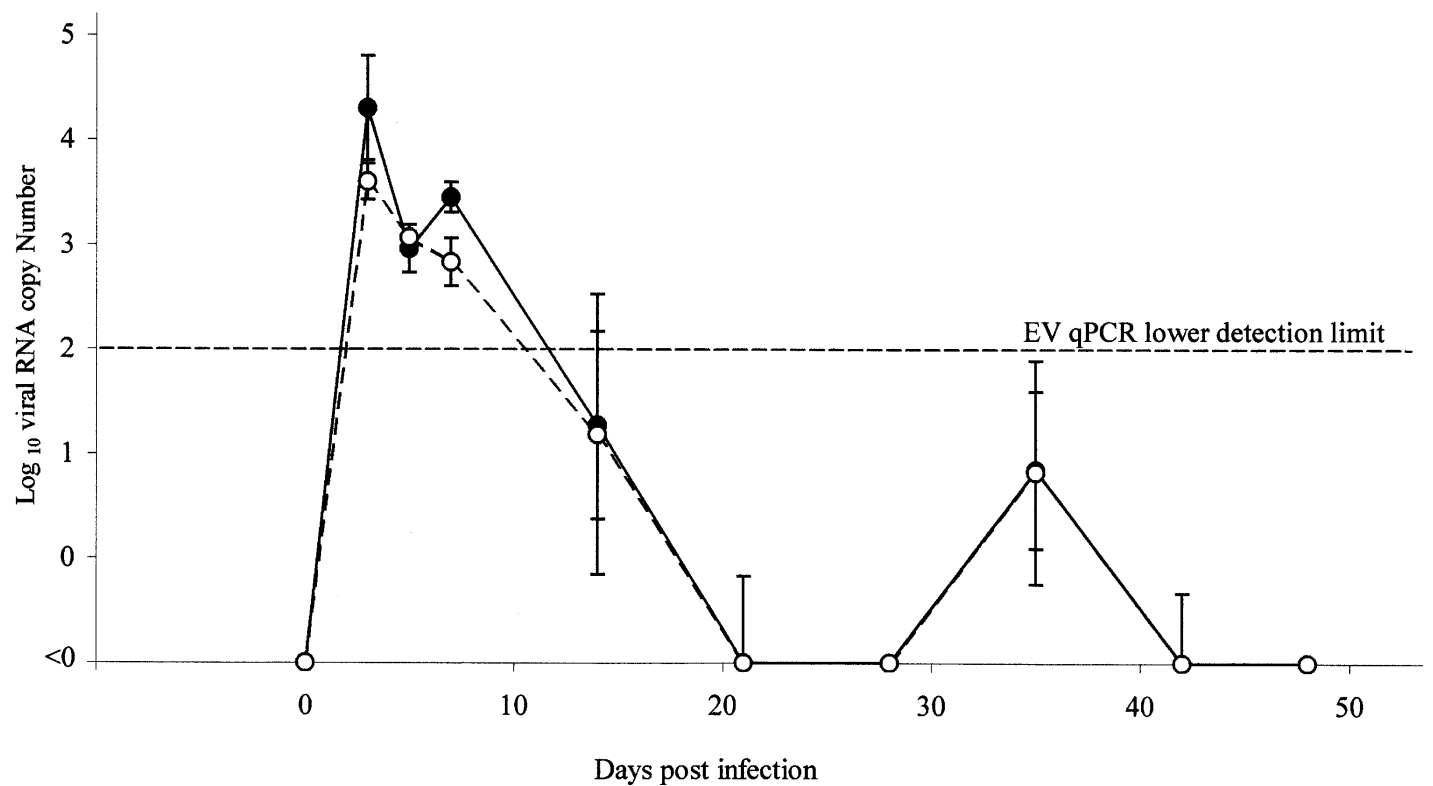

Fig. 3. Mean viral RNA levels in serum $(O)$ and whole blood confidence intervals.

copies (mg tissue) well beyond this time and was still detectable in the hearts of 7 of 14 CVB3-infected mice collected between days 70 and 90 p.i. (Fig. 2). Only a mean daily reduction in RNA copy number of log 0.021 occurred between days 14 and 90 p.i. $\left(r^{2}=0.569\right)$, corresponding to a half-life of $14 \cdot 1$ days. Based on this rate, clearance of viral RNA below the level of detection would have occurred approximately 175 days p.i. Virus infectivity and viral RNA were not detected in control hearts (data not shown).

Viral RNA levels in the blood also peaked on day 3 p.i., but at much lower levels than in the myocardium, and became undetectable after day 14 p.i., except in whole blood from one of four mice collected on day 35 p.i. (Fig. 3). Infectious virus was isolated from whole blood and serum of all four animals tested on days 3 and 5 p.i. and from one of four animals tested on day 7 p.i., but not thereafter (data not shown). Infectious virus or viral RNA was not detected in any samples from uninfected control mice (data not shown).

\section{Persistence of viral RNA in other target organs}

Acute CVB3 infection is a disease which affects multiple organs in mice, although persistent infection is anatomically more restricted at 42 days p.i. (Klingel et al., 1996). To identify possible non-cardiac sites of viral RNA persistence at later stages of infection, the presence of CVB3 RNA was sought using the sensitive EV nPCR assay in a range of tissues collected up to day 90 p.i. Viral RNA was detectable for variable periods in non-cardiac organs and tissues, ranging from 14 to 77 days p.i. (Fig. 4). Viral RNA was detectable only in the myocardium at day 90 p.i. Viral RNA was not detected in tissues from uninfected control mice (data not shown).

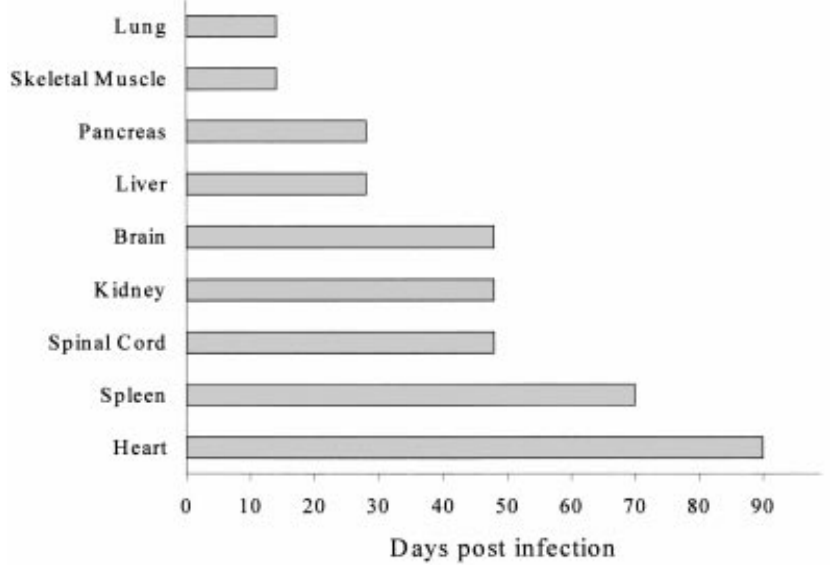

Fig. 4. Maximum duration of viral RNA persistence detected by EV $n P C R$ in organs of CVB3-infected mice.

\section{Relationship between acute phase virus replication and duration of virus persistence in different organs}

To study the kinetics of viral RNA clearance in different organs we quantified mean viral RNA levels in each tissue at day 7 p.i. and sought to correlate this with the duration of virus persistence. We chose to analyse viral RNA levels at day 7 p.i. because viraemic RNA levels were low at this time-point (Fig. 3 ), thus ensuring that viral RNA levels present in the organs studied reflected virus replication rather than viraemic contamination. Pancreatic tissue collected on day 7 p.i. was not available for this analysis. The highest levels of viral RNA were observed in the heart, spleen and lung, with intermediate levels in the brain and kidney, and lower levels in the liver, spinal 


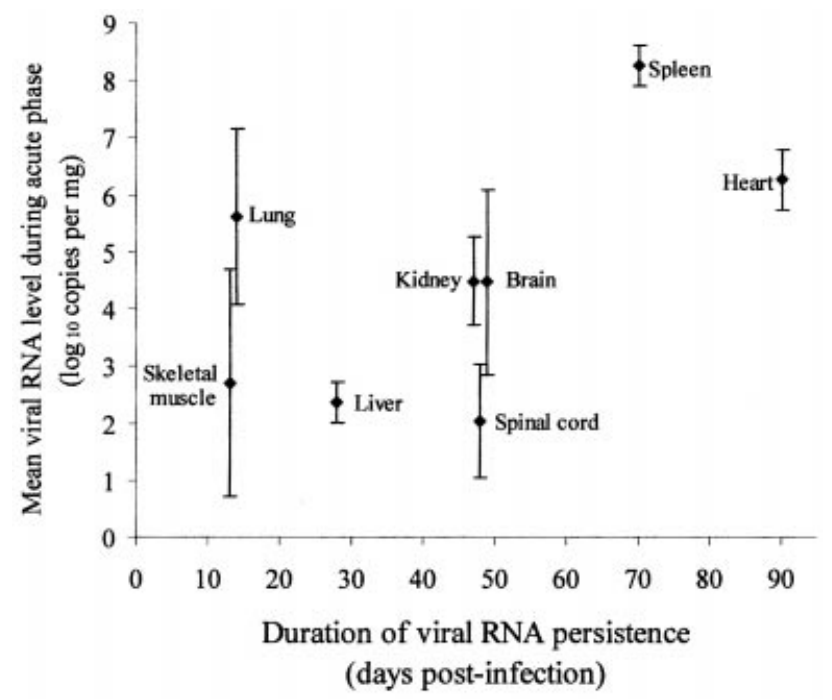

Fig. 5. Relationship between duration of viral RNA persistence and acute phase viral RNA levels in different organs of CVB3-infected mice. Error bars represent $95 \%$ confidence intervals.

cord and skeletal muscle (Fig. 5). The rate of viral RNA clearance in different organs varied considerably, as indicated by the low correlation between acute phase mean viral RNA levels and duration of viral RNA persistence $(r=0 \cdot 479$, $P<0.02$; Spearman's rank correlation).

\section{Discussion}

We have conducted a longitudinal study of viral RNA kinetics in a murine model of CVB3-induced myocarditis. This has yielded valuable insight into the rate of viral RNA clearance during the acute and persistent phases of infection, and its temporal relationship to inflammatory responses. In CVB3-infected immunocompetent mouse strains virus infectivity is rapidly cleared from the heart and other organs. We found that viral RNA was initially cleared at a similar rate. Nevertheless, viral RNA was still present at day 14 p.i. and thereafter the rate of clearance decreased dramatically. Viral RNA persisted in target organs for extended periods and was detected in the hearts from some mice as late as 90 days p.i., by which time myocardial inflammation had resolved.

Several innate and acquired immune effectors have been shown to be important in virus clearance, including NK cell responses (Godeny \& Gauntt, 1987), macrophage-derived inducible nitric oxide synthase (Lowenstein et al., 1996), B cell responses (Mena et al., 1999) and $\mathrm{CD}^{+}$and $\mathrm{CD}^{+} \mathrm{T}$ cell responses (Henke et al., 1995). The reduced clearance rate of viral RNA during the chronic phase of infection suggests that these responses are minimally effective against persistent CVB3 infection. Persisting viral RNA is focally restricted in infected tissues and the level of genomic positive-sense viral RNA is reduced such that equal amounts of genomic- and template-sense RNA are present (Cunningham et al., 1990;
Klingel et al., 1992, 1996). Recently Tam \& Messner (1999) showed that viral RNA persists in a double-stranded form in a murine model of CVBI infection of skeletal muscle. Doublestranded RNA may be resistant to ribonuclease activity and may be associated with reduced viral antigen expression, which would in turn minimize immune-mediated killing of persistently infected cells. Thus, if CVB3 also persists in a double-stranded form, this may account for the long half-life of viral RNA during the chronic phase of infection.

We found considerable variation in the duration of viral RNA persistence in different organs and the time to clearance bore little relation to the initial level of viral RNA. This is consistent with our hypothesis that different mechanisms of virus clearance operate during acute and persistent phases of infection. Previous studies have documented persistence of viral RNA for at least 42 days p.i. in CVB3-induced murine myocarditis (Klingel et al., 1996). In other non-productive picornavirus infections in mice, viral RNA was found to persist from 80 days to 12 months (Destombes et al., 1997; Kyu et al., 1992; See \& Tilles, 1995; Tam et al., 1994). It therefore seems probable that such long-term persistence of viral RNA is not uncommon in picornavirus infections.

The consequences of such low-grade virus persistence for the infected cell or organ are uncertain. Picornavirus replication and gene expression in cultured cells has a profound effect on cellular metabolism and cytoskeletal organization in cultured cells and in the murine heart during acute viral myocarditis (Badorff et al., 1999). The extent of virus replication and genome expression during the persistent enterovirus infection has proved difficult to assess. However, Li et al. (2000) recently identified VPI, the major enterovirus capsid protein, in myocardial tissue from $54.5 \%$ of patients with myocarditis and $37.5 \%$ with dilated cardiomyopathy by employing improved immunohistochemical detection methods. It will be interesting to apply these methods to the study of murine models of persistent enterovirus infection. There is currently some debate as to whether immune responses in chronic murine myocarditis are directed against viral antigens or host myocardial determinants. The persistence of both viral RNA and viral antigen raises the possibility that immune responses may target viral or virus-induced antigens.

Since enterovirus persistence may be established without the selection of replication-defective mutants (Tam \& Messner, 1999), it is theoretically possible that productive infection might resume under appropriate conditions. The persistence of non-productive infection beyond the resolution of myocardial inflammation in the present study indicates that the inflammatory response is not required to contain virus persistence. However, Shioi et al. (1996) found increased cytokine expression and localization in the myocardium even during the post-inflammatory phase of encephalomyocarditis virusinduced murine myocarditis. It will clearly be important to determine the role of these chronic immune responses in both virus persistence and ongoing cardiac pathology. 
We previously suggested that virus persistence in noncardiac tissues might act as a reservoir of antigenic stimulation, which could explain the persistence of enterovirus-specific $\operatorname{IgM}$ and $\operatorname{Ig} \mathrm{A}$ responses in patients with chronic heart disease (Muir et al., 1989). Klingel et al. (1996) found that the spleen and the lymph nodes were major sites of extracardiac persistence in murine CVB3-induced myocarditis at 42 days p.i. and suggested that this may play a role in the maintenance of chronic disease. In the present study, clearance of viral RNA from spleen was observed at about day 77 p.i. It is interesting to note that clearance of virus from the spleen coincided with resolution of myocardial inflammation. Anderson et al. (1996) showed that localization of viral RNA in splenic germinal centres only occurred in mouse strains that are susceptible to myocarditis. Taken together, these observations suggest that virus replication or retention in follicular germinal centres of the spleen drives the immune response that gives rise to inflammation of the myocardium and other virus-infected organs.

Although the concept that enteroviruses may persist in the immunocompetent host challenges current understanding of enterovirus biology, the findings presented here, together with other recent data, indicate that the enterovirus RNA persistence is a true biological phenomenon characterized by a reduced rate of clearance of the viral genome and is not an artefact caused by the greater sensitivity of genome detection methods relative to infectivity assays. Future studies should aim to define further the molecular basis and biochemical consequences of enterovirus persistence, to identify the antigenic stimulus that drives the inflammatory response in enterovirus-induced myocardial disease and to clarify further the role of immune effectors in virus clearance, persistence and immunopathology.

This paper is dedicated to the memory of Hugh Faulkner, co-founder and first honorary director of the Chronic Fatigue Syndrome Research Foundation (originally founded as the Persistent Virus Disease Research Foundation)

This study was funded by the CFS Research Foundation. K.N.R. is the recipient of a scholarship from the Association of Commonwealth Universities. S. J.I. is supported by the Philip Fleming Trust. We thank the staff of the Biological Research Services Facility, Rayne Institute, St Thomas' Campus, King's College London, for invaluable technical assistance and Dr J. Cason for useful discussions during preparation of this manuscript.

\section{References}

Anderson, D. R., Wilson, J. E., Carthy, C. M., Yang, D., Kandolf, R. \& McManus, B. M. (1996). Direct interactions of coxsackievirus B3 with immune cells in the splenic compartment of mice susceptible or resistant to myocarditis. Journal of Virology 70, 4632-4645.

Badorff, C., Lee, G.-H., Lamphear, B. J., Martone, M. E., Campbell, K. P., Rhoads, R. E. \& Knowlton, K. U. (1999). Enteroviral protease 2A cleaves dystrophin: evidence of cytoskeletal disruption in an acquired cardiomyopathy. Nature Medicine 5, 320-326.
Cunningham, L., Bowles, N. E., Lane, R. J. M., Dubowitz, V. \& Archard, L. C. (1990). Persistence of enteroviral RNA in chronic fatigue syndrome is associated with the abnormal production of equal amounts of positive and negative strands of enteroviral RNA. Journal of General Virology 71, 1399-1402.

Destombes, J., Couderc, T., Thiesson, D., Girard, S., Wilt, S. G. \& Blondel, B. (1997). Persistent poliovirus infection in mouse motorneurons. Journal of Virology 71, 1621-1628.

Godeny, E. K. \& Gauntt, C. J. (1987). Murine natural killer cells limit coxsackievirus B3 replication. Journal of Immunology 139, 913-918.

Grodums, E. I. \& Dempster, G. (1959). Myocarditis in experimental coxsackie B3 infection. Canadian Journal of Microbiology 5, 605-615.

Henke, A., Huber, S., Stelzner, A. \& Whitton, J. L. (1995). The role of $\mathrm{CD}^{+} \mathrm{T}$ lymphocytes in coxsackievirus B3-induced myocarditis. Journal of Virology 69, 6720-6728.

Jubelt, B., Gallez-Hawkins, G., Narayan, O. \& Johnson, R. T. (1980). Pathogenesis of human poliovirus infection in mice. I. Clinical and pathological studies. Journal of Neuropathology \& Experimental Neurology 39, 138-148.

Kandolf, R. \& Hofschneider, P. H. (1985). Molecular cloning of the genome of a cardiotropic coxsackie B3 virus: full length reversetranscribed recombinant CDNA generates infectious virus in mammalian cells. Proceedings of the National Academy of Sciences, USA 82, 4818-4822.

Klingel, K., Hoenadl, C., Canu, A., Albrecht, M., Seemann, M., Mall, G. \& Kandolf, R. (1992). Ongoing enterovirus-induced myocarditis is associated with persistent heart muscle infection: quantitative analysis of virus replication, tissue damage and inflammation. Proceedings of the National Academy of Sciences, USA 89, 314-318.

Klingel, K., Stephan, S., Sauter, M., Zell, R., McManus, B. M., Bultmann, B. \& Kandolf, R. (1996). Pathogenesis of murine enterovirus myocarditis: virus dissemination and immune cell targets. Journal of Virology 70, 8888-8895.

Kyu, B., Matsumori, A., Sato, Y., Okada, Y., Chapman, N. \& Tracy, S. (1992). Cardiac persistence of cardioviral RNA detected by polymerase chain reaction in a murine model of dilated cardiomyopathy. Circulation 86, 522-530.

Li, Y., Bourlet, T., Andreoletti, L., Mosnier, J.-F., Peng, T., Yang, Y., Archard, L. C., Pozzetto, B. \& Zhang, H. (2000). Enteroviral capsid protein VPI is present in myocardial tissues from some patients with myocarditis or dilated cardiomyopathy. Circulation 101, 231-234.

Lipton, H. L. (1975). Theiler's virus infection in mice: an unusual biphasic disease process leading to demyelination. Infection and Immunity 11, 1147-1155.

Lipton, H. L. \& Dal Canto, M. C. (1976). Chronic neurological disease in Theiler's virus infection of SJL/J mice. Journal of Neurological Sciences 34, 201-207.

Lowenstein, C. J., Hill, S. L., Lafond-Walker, A., Wu, J., Allen, G., Landavere, M., Rose, N. R. \& Herskowitz, A. (1996). Nitric oxide inhibits viral replication in murine myocarditis. Journal of Clinical Investigation 97, 1837-1843.

McKinney, R. E., Katz, S. L. \& Wilfert, C. M. (1987). Chronic enteroviral meningoencephalitis in agammaglobulinemic patients. Reviews of Infectious Diseases 9, 334-356.

Melchers, W., Zoll, J., van Kuppeveld, F., Swanink, C. \& Galama, J. (1994). There is no evidence for persistent enterovirus infections in chronic medical conditions in humans. Reviews of Medical Virology 4, 235-243.

Mena, I., Perry, C. M., Harkin, S., Rodriguez, F., Gebhard, J. \& Whitton, J. L. (1999). The role of B lymphocytes in coxsackievirus B3 infection. American Journal of Pathology 155, 1205-1215. 
Miller, J. R. (1981). Prolonged intracerebral infection with poliovirus in asymptomatic mice. Annals of Neurology 9, 590-596.

Modlin, J. F. (2000). Coxsackieviruses, echoviruses, and newer enteroviruses. In Mandell, Douglas \& Bennett's Principles \& Practice of Infectious Diseases, vol. 2, 5th edn, pp. 1904-1919. Edited by G. L. Mandell, J. E. R. Bennett \& R. Dolin. Philadelphia: Churchill Livingstone.

Muir, P. \& Archard, L. C. (1994). There is evidence for persistent enterovirus infections in chronic medical conditions in humans. Reviews in Medical Virology 4, 245-250.

Muir, P., Nicholson, F., Tilzey, A. J., Signy, M., English, T. A. H. \& Banatvala, J. E. (1989). Chronic relapsing pericarditis and dilated cardiomyopathy: serological evidence of persistent enterovirus infection. Lancet i, 804-807.

Nicholson, F., Meetoo, G., Aiyar, S., Banatvala, J. E. \& Muir, P. (1994). Detection of enterovirus RNA in clinical samples by nested polymerase chain reaction for rapid diagnosis of enterovirus infection. Journal of Virological Methods 48, 155-166.

Reed, L. J. \& Muench, H. (1938). A simple method of estimating fifty per cent endpoints. American Journal of Hygiene 27, 493-497.

Reetoo, K. N., Osman, S. A., Illavia, S. J., Banatvala, J. E. \& Muir, P. (1999). Development and evaluation of quantitative-competitive PCR for quantitation of coxsackievirus B3 RNA in experimentally infected murine tissues. Journal of Virological Methods 82, 145-156.

Ren, R. B., Costantini, F., Gorgacz, E. J., Lee, J. J. \& Racaniello, V. R. (1991). Transgenic mice expressing a human poliovirus receptor: a new model for poliomyelitis. Cell 63, 353-362.

See, D. M. \& Tilles, J. G. (1995). Pathogenesis of virus-induced diabetes in mice. Journal of Infectious Diseases 171, 1131-1138.

Shioi, T., Matsumori, A. \& Sasayama, S. (1996). Persistent expression of cytokine in the chronic stage of viral myocarditis in mice. Circulation 94, 2930-2937.

Strongwater, S. L., Dorivini-Zis, K., Ball, R. D. \& Schnitzer, T. J.
(1984). A murine model of polymyositis-induced by coxsackievirus BI (Tucson strain). Arthritis and Rheumatism 27, 422-433.

Tam, P. E. \& Messner, R. P. (1999). Molecular mechanisms of coxsackievirus persistence in chronic inflammatory myopathy: viral RNA persists through formation of a double-stranded complex without associated genomic mutations or evolution. Journal of Virology 73, 10113-10121.

Tam, P. E., Schmidt, A. M., Ytterberg, S. R. \& Messner, R. P. (1991). Viral persistence during the developmental phase of coxsackie B1induced murine polymyositis. Journal of Virology 65, 6654-6660.

Tam, P. E., Schmidt, A. M., Ytterberg, S. R. \& Messner, R. P. (1994). Duration of virus persistence and its relationship to inflammation in the chronic phase of coxsackievirus B1-induced murine polymyositis. Journal of Laboratory and Clinical Medicine 123, 346-356.

Webb, S. R., Loria, R. M., Madge, G. E. \& Kibrick, S. (1976). Susceptibility of mice to group B coxsackievirus is influenced by the diabetic gene. Journal of Experimental Medicine 143, 1239-1248.

Woodruff, J. F. \& Kilbourne, E. D. (1970). The influence of quantitated post-weaning undernutrition on coxsackievirus B3 infection of adult mice. I. Viral persistence and increased severity of lesions. Journal of Infectious Diseases 121, 137-163.

Yoon, J. W., Onodera, T. \& Notkins, A. L. (1978). Virus-induced diabetes mellitus. XV. Beta cell damage and insulin-dependent hyperglycaemia in mice infected with coxsackie virus B4. Journal of Experimental Medicine 148, 1068-1080.

Zhang, H., Yousef, G. E., Ouyang, X. \& Archard, L. C. (1994). Characterization of a murine model of myocarditis induced by a reactivated coxsackievirus B3. International Journal of Experimental Pathology 75, 99-110.

Received 16 June 2000; Accepted 3 August 2000 